\title{
Osteotomien am Fußskelett nach in Achsenabweichung verheilten Frakturen
}

\author{
H. Zwipp, C. Dahlen, J. M. Gavlik
}

\section{Zusammenfassung}

„Osteotomien“ am Talus, auch bei fehlverheilter intraartikulärer Fraktur, sind möglich und der Arthrodese vorzuziehen. Osteotomien zur Achsenkorrektur am Calcaneus sind bei extraartikulärer Fraktur selten, bei fehlverheilten Luxationsfrakturen neben der subtalaren Fusion in der Regel zwingend notwendig, um eine biomechanische Reorientierung des Rückfußes zu erzielen. Korrekturosteotomien mit achsenkorrigierender Spaninterposition im Chopart-/Lisfranc-Bereich dienen vor allem der Bilanzierung von lateraler und medialer Fußsäule. Achsenkorrigierende Osteotomien der Metatarsalia sind zur Beseitigung von Metatarsalgien sinnvolle Eingriffe. Bei notwendigen Korrekturen komplexer Fehlstellungen bietet die 3DPC-Planung eine neue Dimension präoperativer Planung.

Osteotomien nach in Achsenabweichung verheilten Frakturen am Fuß- skelett sind seltene Indikationen. Dies liegt daran, dass achsenabweichende Frakturen des Talus in praxi immer, die des Calcaneus in $87 \%$ der Fälle mit einer Gelenkfraktur assoziiert sind [4] und somit in der Regel eine reorientierende Arthrodese auf einer oder mehreren Ebenen erfordern. Ähnliches gilt auch für das Os naviculare, das Os cuboideum und die Cuneiformia. Die einzigen Schaftfrakturen am Fußskelett stellen die diaphysären Metatarsalia-Brüche dar, welche nur selten, auch bei konservativer Behandlung eine korrekturbedürftige Achsenabweichung zurücklassen. Letztere ist am ehesten noch im subcapitalen Bereich mit gelegentlich grotesker Abweichung der Metatarsaliaköpfchen beobachtbar. Dennoch gibt es am Fußskelett Indikationen zur Osteotomie, die im Folgenden aufgezeigt werden sollen.

\section{Talus}

Wenn eine sukzessive Auflösung einer fehlverheilten Fraktur des Talus im Bereich der ehemaligen Fraktur mit Hilfe von kleinen Meiseln, scharfen Löffeln und feinem Luer bis zur vollständigen Separation der zwei inkongruent verheilten Fragmente als „Osteotomie“ bezeichnet werden kann, so gibt es diese auch am Talus (Abb.1). Bei den fast ausnahmslos intraartikulären, zentralen Frakturen des Talus genügt allerdings nicht die Osteoto-

OP-JOURNAL 2000; 16: 72-78

(c) Georg Thieme Verlag Stuttgart · New York mie zur biomechanischen Reorientierung aus, sondern ist immer mit einer Gelenklösung des Talus im oberen, subtalaren oder talo-navikularen Gelenkabschnitt zur korrekten Achsenausrichtung notwendigerweise verbunden. Die „Osteotomie“ des Talus ist in der Regel nur über einen bilateralen Zugang möglich, im Falle einer fehlverheilten Fraktur im Corpus tali nur mit zusätzlicher Osteotomie des Innenknöchels. CT und MRT coronar und axial sind zur präoperativen Planung notwendig. Bei einer pantalaren Mobilisation ist die Gefahr einer postoperativen Osteonekrose groß und dem Patienten präoperativ unbedingt mitzuteilen. Diese extrem anspruchsvollen Rekonstruktionseingriffe sollten immer dem Patienten im Sinne eines Rekonstruktionsversuches als alternative Möglichkeit zur definitiven Arthrodese dargestellt werden. Die eigenen fünf erfolgreichen Rekonstruktionen geben allerdings Anlass, dieses hochgesteckte Ziel weiterhin zu verfolgen.

\section{Calcaneus}

Erhebliche Rückfußachsenfehlstellungen nach Calcaneusfraktur ohne Gelenkbeteiligung sind eine Ausnahme (Abb.2). Daher sind reorientierende Calcaneusosteotomien, die die fehlverheilte Bruchzone wieder auflösen, meist mit einer subtalaren Arthrodese zu kombinieren. Diese oft grotesken Fehlverheilungen sind in der Regel auf übersehene oder konservativ behandelte Luxationsfrakturen des Calcaneus zurückzuführen (Abb.3). Eine alleinige subtalare Arthrodese könnte in diesen Fällen die schwergestörte Biomechanik des Rückfußes nicht beseitigen und muss zwangsläufig zu einem unbefriedigenden Resultat führen [2,4]. Dies deshalb, da Peronealsehnenimpingement, Abutement, Rückfußachsenfehlstellungen und dynamische Muskelimbalancen unberücksichtigt blieben. Auf Grund der Komplexität der Frakturpathologie ist in diesen Fällen zur Operationsplanung ein dreidimensionelles Kunststoffmodell (Abb.4) zur „Voroperation“ notwendig oder neuerdings die 3D-PCPlanung (Abb.3). Calcaneusosteotomien als notwendige operative Maßnahme in der Verbindung mit einer reorientierenden subtalaren Arthrodese erfordern ein hohes Ausmaß an präoperativer Planung, atraumatischer OP-Technik und operativer Erfahrung in der rekonstruktiven Fußchirurgie. Dies insbesondere deswegen, da für die meisten CalcaneusOsteotomien ein zusätzlicher medialer Zugang zur Schonung des neurovasculären Bündels unumgänglich ist. Die Ergebnisse in acht eigenen Fällen ermutigen, dieses Konzept weiter zu verfolgen. Die jetzt mögliche 3D-PC-Planung [1] könnte geeignet sein, „sogenannte Lernkurven“ $\mathrm{zu}$ verkürzen und $\mathrm{zu}$ verbessern. 

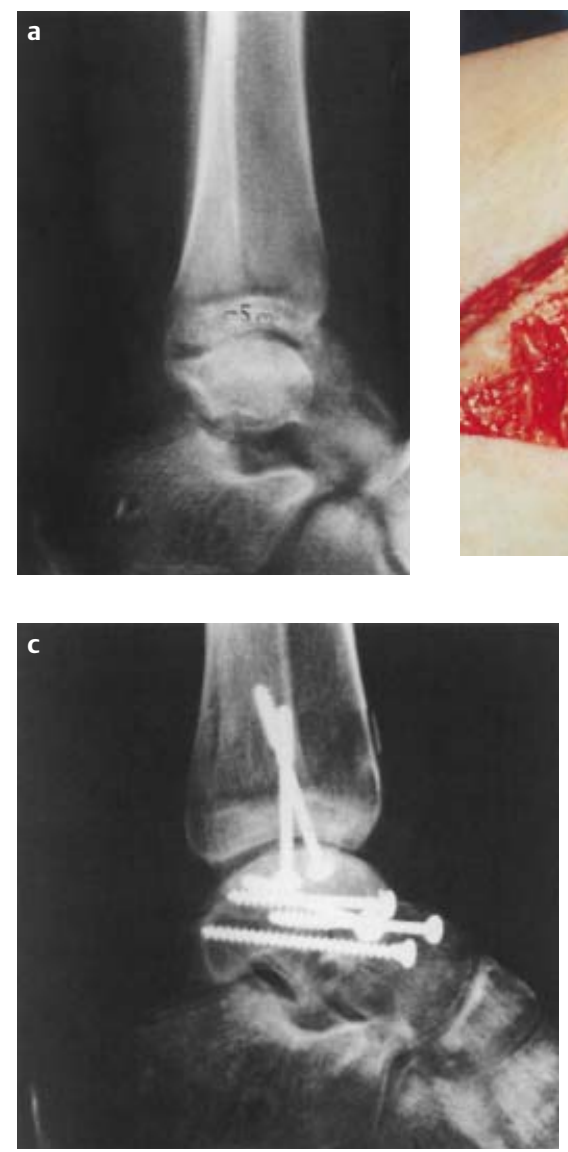

Abb.1 „Osteotomie des Talus“ bei einem 17jährigen Patienten, fünf Monate nach extremer Fehlverheilung einer mit Fixateur externe behandelten Taluskörperluxationsfraktur. (a) Die seitliche OSG-Aufnahme zeigt die Fehlverheilung des Talus mit fast $10 \mathrm{~mm}$ Stufenbildung in der vorderen Trochlea tali, die eine Dorsalflexion verhindert. Auch das SubtalarGelenk ist durch die Kippung der Trochlea tali nach plantar und die zusätzliche Innenrotation des Talus erheblich inkongruent erkennbar. (b) Durch einen bilateralen Zugang, hier mit zusätzlicher Osteotomie des Innenknöchels (IK) kann die fehlverheilte Frakturfläche ( $\mathrm{T} 1$ = dorso-mediale Trochlea, $\mathrm{T} 2$ = antero-mediale Trochlea) dargestellt und sukzessive mit kleinem Meißel und feinen Löffeln wieder aufgelöst werden. Innenrotationsfehlstellung des Taluskopfes (TK). (c) Bereits intraoperativ ist die Kongruenz des Talus wiederhergestellt. Eine funktionelle Nachbehandlung kann erfolgen. (d) Auch drei Jahre nach „Osteotomie des Talus“ ist keine Partialnekrose erkennbar. Gute Funktion. Beschwerdefreiheit.
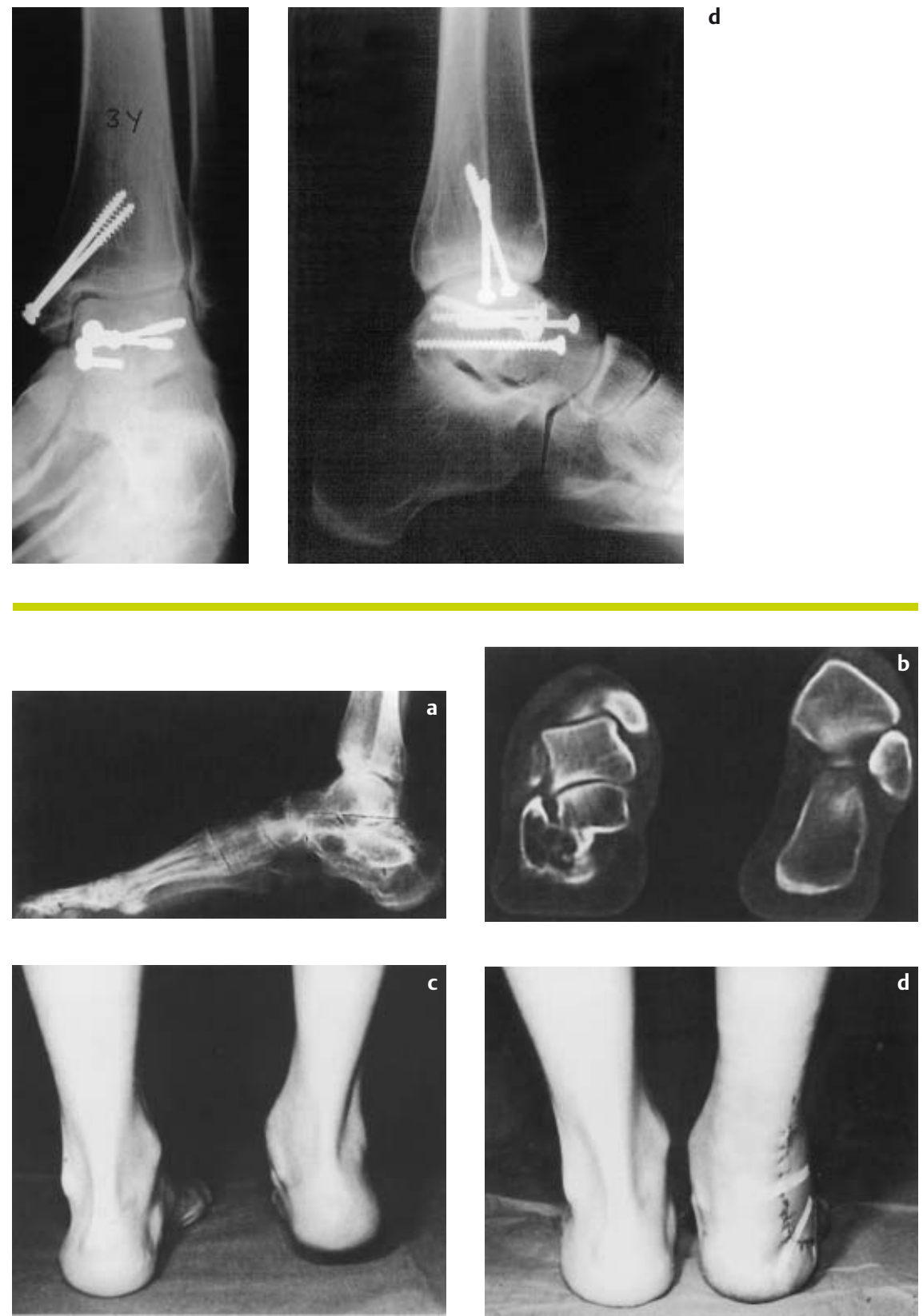

Abb. 2 Legende s. S. 74 


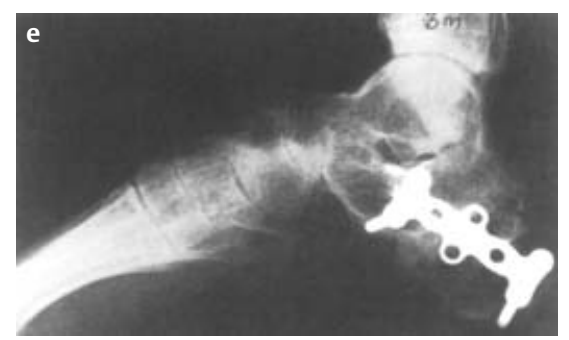

Abb. 2 Beispiel einer extrartikulären Fersenbeinkorrektur nach erheblicher CalcaneusFehlverheilung (18 Monate) bei medialseitig zweitgradig offener, extraartikulärer Fersenbeinfraktur und konservativer auswärtiger Behandlung einer 25jährigen Patientin. Die gelernte Kellnerin ist bei erheblich gestörter Rückfußstatik berufs- und arbeitsunfähig. (a) Die präoperative Belastungsaufnahme seitlich zeigt den erheblich abgeflachten Tubergelenkwinkel. (b) Erst das coronare CT lässt die durchgemachte Frakturpathologie richtig erkennen. Dabei wird deutlich, dass bei der seinerzeit extraartikulären Fraktur das tuberositäre Hauptfragment gegenüber dem sustentacularen Fragment weit nach lateral disloziert verblieben ist und somit zur Höhenminderung des Rückfußes, Verkürzung und Verbreiterung des Calcaneus mit intakter posteriorer Facette führte. (c) Der klinische Aspekt von hinten lässt den Rückfußvalgus mit Lateralversetzung gut erkennen. Durch den Höhenverlust kann der Rückfuß die Körperlast nicht aufnehmen, ein physiologischer Abrollvorgang durch die zwischenzeitlich eingetretene Vorfußeinsteifung ist nicht möglich. (d) Der klinische Aspekt bereits 8 Tage nach dem Eingriff zeigt die Achsenkorrektur und das physiologische Auffußen des Rückfußes. (e) Aufgrund des intakten Subtalar-Gelenkes wird lediglich die Rückfußkorrektur über einen lateralen Zugang mit Osteotomie und autologer kortiko-spongiöser Spaninterposition mit stabiler Plättchenosteosynthese angestrebt, der laterale Bulge wird reseziert, eine früh-funktionelle Behandlung ist möglich. Bereits nach 3 Monaten ist der Rückfuß konsolidiert und schmerzfrei voll belastbar.
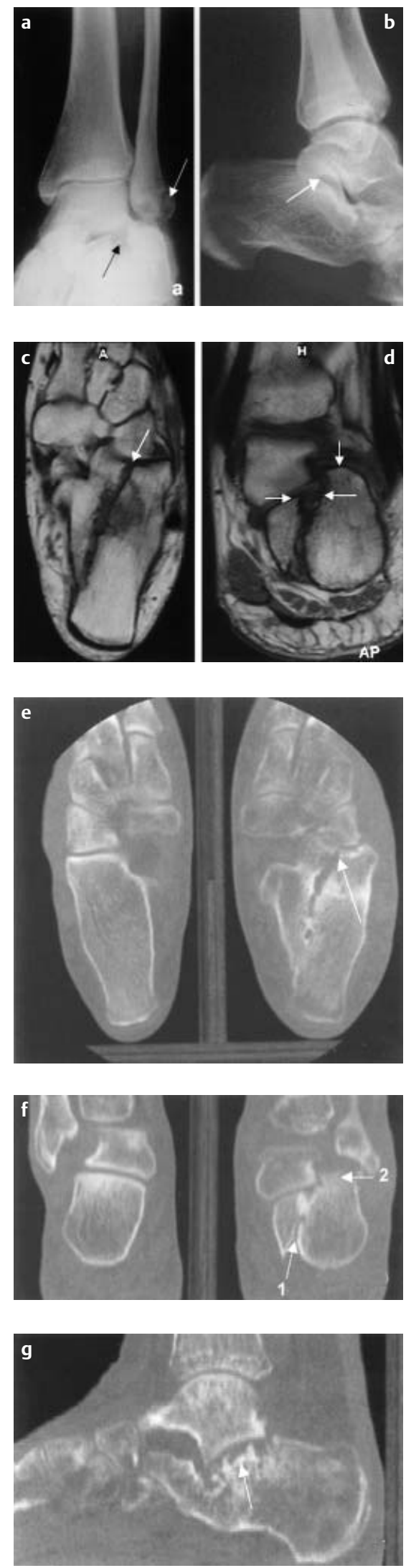

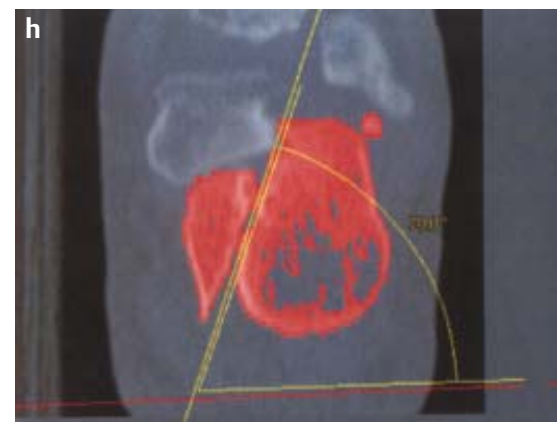

Abb. 3 Übersehene und fehlverheilte Calcaneus-Luxationsfraktur links einer 34jährigen Frau, die wegen anhaltender Beschwerden nach sekundärer, weiterführender Diagnostik eine Calcaneusosteotomie zur Reorientierung des Rückfußes verbunden mit subtalarer Arthrodese erhalten musste. (a) Die a. p.-Projektion des linken OSG vom Unfalltag zeigt den knöchernen Ausriss des proximalen Peronealsehnenretinaculums (weißer Pfeil), der als Weber-A-Fraktur fehlgedeutet wurde, wenngleich der weite Spalt im Subtalar-Gelenk (schwarzer Pfeil) erkennbar ist. (b) Die seitliche Rückfußaufnahme zeigt die suspekte Doppelkonturierung der posterioren Facette (weißer Pfeil). (c) Erst die Kernspinuntersuchung 6 Monate nach dem Trauma, die wegen anhaltender, unerklärter Beschwerden veranlasst wurde, zeigt die extreme Subluxationsstellung des gesamten lateralen Fersenbeinkörpers nach initialem Spaltbruch, durch die posteriore Facette bis in das Calcaneo-Cuboid-Gelenk hinein verlaufend. (d) Die coronare Schicht zeigt die weite Versetzung des Corpus calcanei mit posteriorer Facette nach lateral bis unter den Außenknöchel. (e) Das axiale CT zeigt die partielle Verheilung des Spaltbruches im dorsalen und plantaren Bereich. Zum Calcaneo-Cuboid-Gelenk (weißer Pfeil) erscheint die Fraktur nicht fest. (f) Der coronare CT-Schnitt zeigt den relativ kleinen sustentacularen Fragmentanteil der partiell [1] mit dem großen Tuberfragment fehlverheilt ist sowie den Höhenversatz der posterioren Facette bis unter die Fibula, weit nach lateral subluxiert [2]. (g) Die sagittale Rekonstruktion zeigt die zwischenzeitlich eingetretene Zerstörung des Subtalar-Gelenkes (weißer Pfeil). (h) Die im PC dargestellten, übermittelten Daten der Computertomographie zeigen in der 2D-Rekonstruktion die notwendige Osteotomie-Ebene von 70 Grad gegenüber der Plantarebene. (i) In der Transversalebene ergibt sich ein Osteotomiewinkel von 25,9 Grad gegenüber der anatomischen Fußachse. (j) Die postoperative OSG-a. p.-Aufnahme nach Calcaneusosteotomie und subtalarer Fusion zeigt jetzt nach Medialisierung des Corpus calcanei wieder den freien Bereich unterhalb der Fibula (Pfeil), das Abutement ist vollständig beseitigt. (k) Die postoperative axiale Calcaneusprojektion zeigt die korrekte Rückfußachse bei jetzt geschlossenem Frakturspalt. (I) Die seitliche Fußaufnahme zeigt die annähernde Wiederherstellung des Böhlerwinkels von jetzt 33 Grad gegenüber der gesunden Seite mit 36 Grad. (m) Die sagittale postoperative CT-Re- 

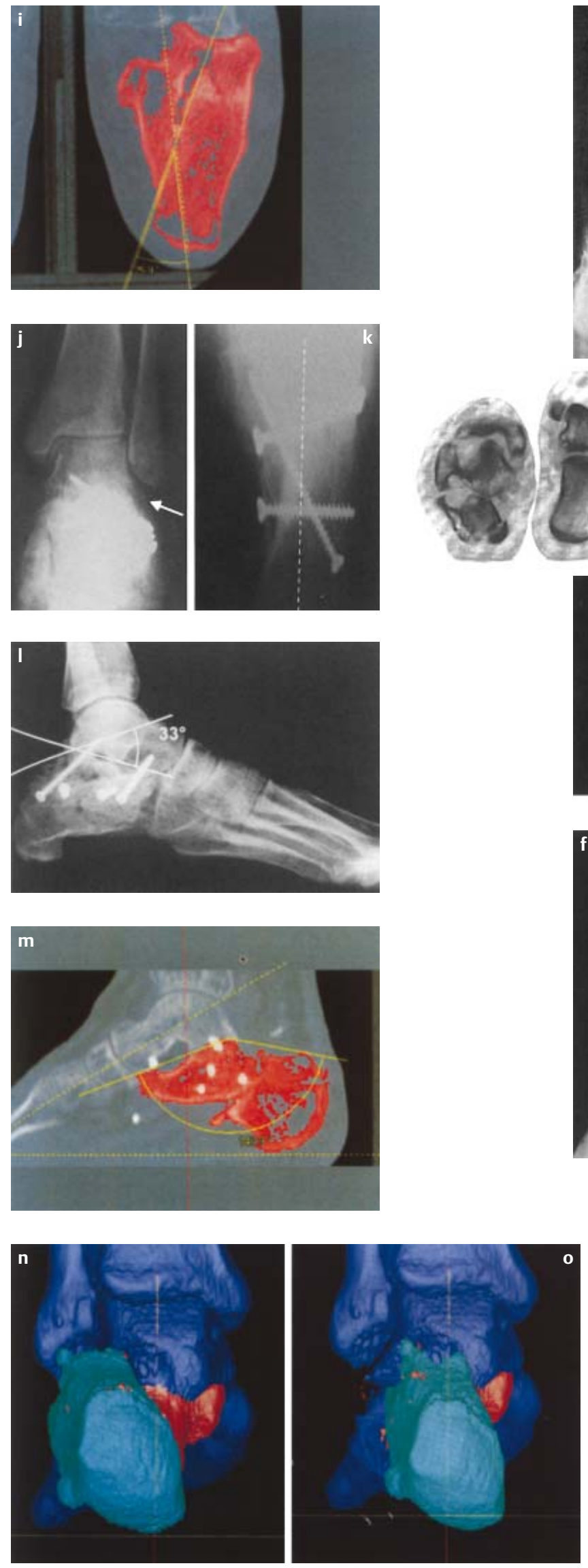

4 Legende Abb. 3 (Fortsetzung)

konstruktion zeigt die jetzt physiologische talo-metatarsale Achse sowie den von 165 Grad auf 147,9 verbesserten reziproken Böhlerwinkel (Sollwert der gesunden Seite $144 \mathrm{Grad}$ ). (n, o) Die 3D-Visualisierung präoperativ (n) zeigt sehr deutlich die Wiederherstellung der physiologischen Rückfußachse postoperativ (o). 
Abb. 4 Komplexe OSG- und Rückfußrekonstruktion mit subtalarer Arthrodese bei einem 23jährigen Patienten 4 Monate nach Calcaneus-Luxationsfraktur. Bei dem seinerzeit polytraumatisierten Patienten war bei konservativer Behandlung des Fersenbeinbruches offensichtlich die Subluxation im OSG nicht erkannt worden, die jetzt nach Konsolidierung der Fersenbeinfraktur zur erheblichen Schmerzhaftigkeit besonders im OSG geführt hat. (a) Die seitliche Fußbelastungsaufnahme zeigt den Kollaps des gesamten Rückfußes im Sinne des posttraumatischen Plattfußes. (b) Erst die Computertomographie (links) und ein danach geschnittenes dreidimensionales Modell (rechts) lassen die massiven Traumafolgen gut erkennen. Besonders durch das Modell wird die präoperative Planung erleichtert. (d-f) Über drei Zugänge: anterior zum OSG, ausgedehnt lateral und medial zum Fersenbein gelingt es den tief impaktierten, nach lateral gekippten und bereits im Calcaneus eingewachsenen Talus wieder zu heben, wobei der Knorpel der Facies lateralis tali nach 4 Monaten noch erstaunlich gut erhalten ist. (d) Durch die von medial-plantar nach lateral-dorsal korrigierende Osteotomie und entsprechende autologe kortiko-spongiöse Spaninterposition zwischen Talus und posteriorer Facette kann der Rückfuß zur Positionierung des Talus im OSG entsprechend unterfüttert und im Sinne der korrigierenden Schraubenarthrodese stabilisiert werden. (e) 1 Jahr nach der Rekonstruktion ist der Talus kongruent in der OSG-Gabel, der Patient schmerzfrei in seinem Beruf als Klempner arbeitsfähig. Die OSG-Beweglichkeit beträgt $0 / 0 / 30$ Grad. (f) Nach 5 Jahren ist kaum eine Arthrose im OSG nachweisbar, die plantare Schraube zwischenzeitlich entfernt. Der Patient ist voll arbeitsfähig.
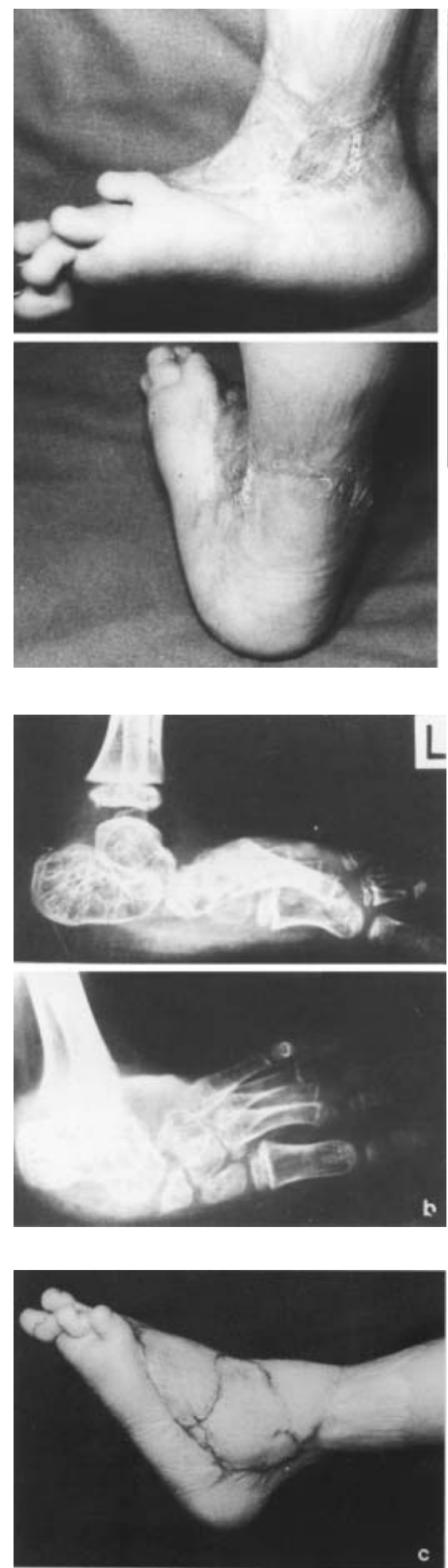

Abb.5 Beispiel zur Bedeutung der plastischen Chirurgie in der erweiterten Fußchirurgie. (a, b): Komplexe Fußdeformität nach indirektem und direktem Trauma eines seinerzeit 6jährigen türkischen Knaben, der im Rahmen eines Erdbebens eine schwere Quetschverletzung des linken Fußes sowie Verbrennungen der Haut erlitt. (c, d) Bei dem jetzt 10jährigen Knaben werden die kontrakten Narben im Sprunggelenks- und Fußbereich entfernt und vitales Gewebe mittels freiem M. latissi-

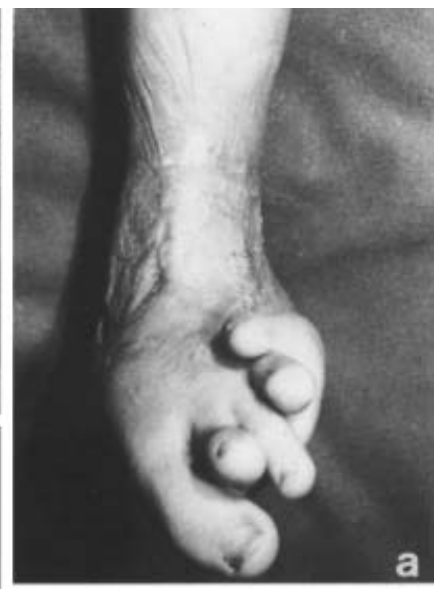

d
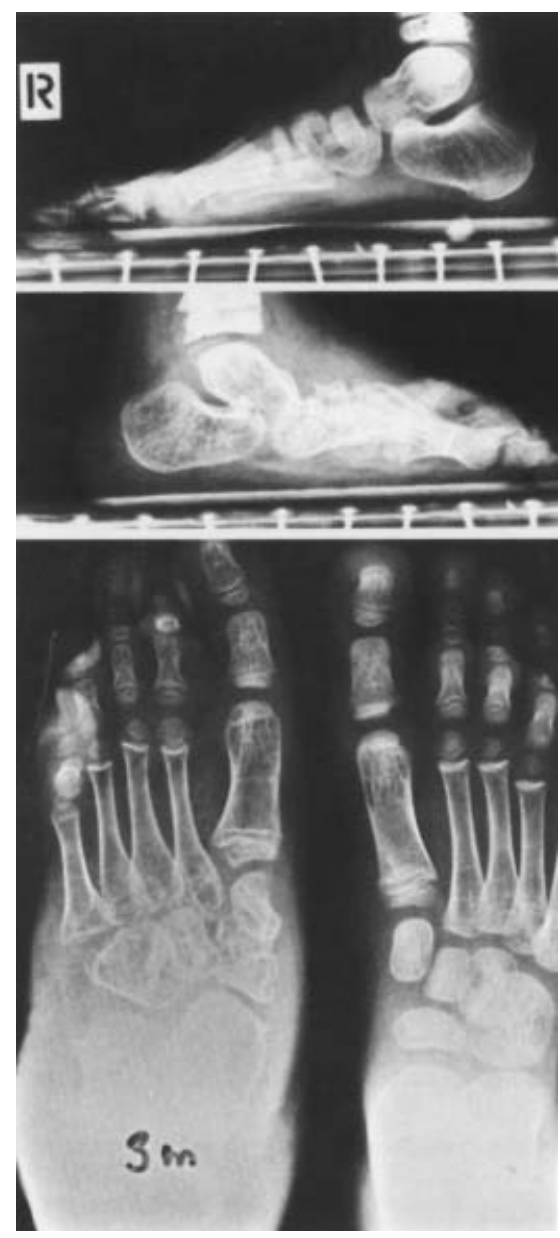

mus dorsi in gleicher Sitzung ersetzt. Korrekturosteotomie des Calcaneus mit autologer Spaninterposition zur Verlängerung der lateralen Fußsäule im Sinne der Evans-Osteotomie. Das autologe kortiko-spongiöse Material hierzu wird von der subtraktiven Osteotomie der Cuneiformia verwandt. Deutliche Verbesserung der Weichteile und des Fußwurzelskelettes bei der Dreimonatskontrolle. Zehen- und Vorfußkorrektur sollten in 2. Sitzung erfolgen. 

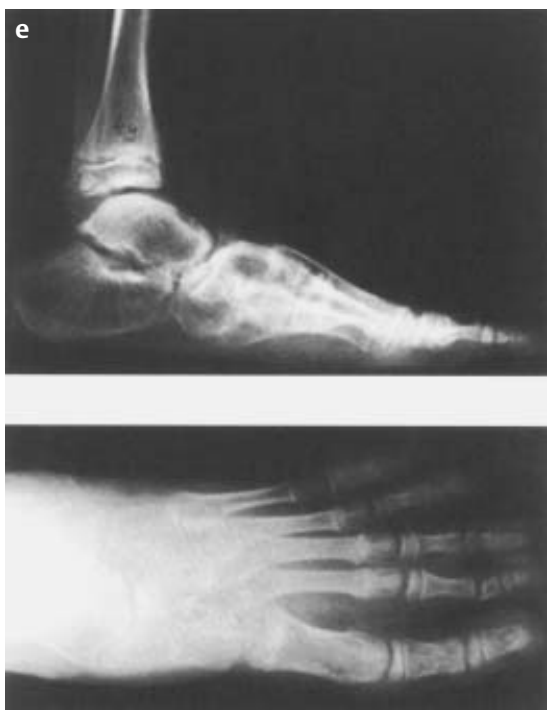

Abb.5e,f Fünf Jahre später zeigt der jetzt 14jährige Patient einen mehr oder weniger normalen Fuß ohne notwendige Nachkorrektur des Vorfußes, d.h. spontane Normalentwicklung des Fußes nach plastischer Deckung und Fußwurzelkorrektur. Der Junge ist voll sportfähig und kann normales Schuhwerk tragen.
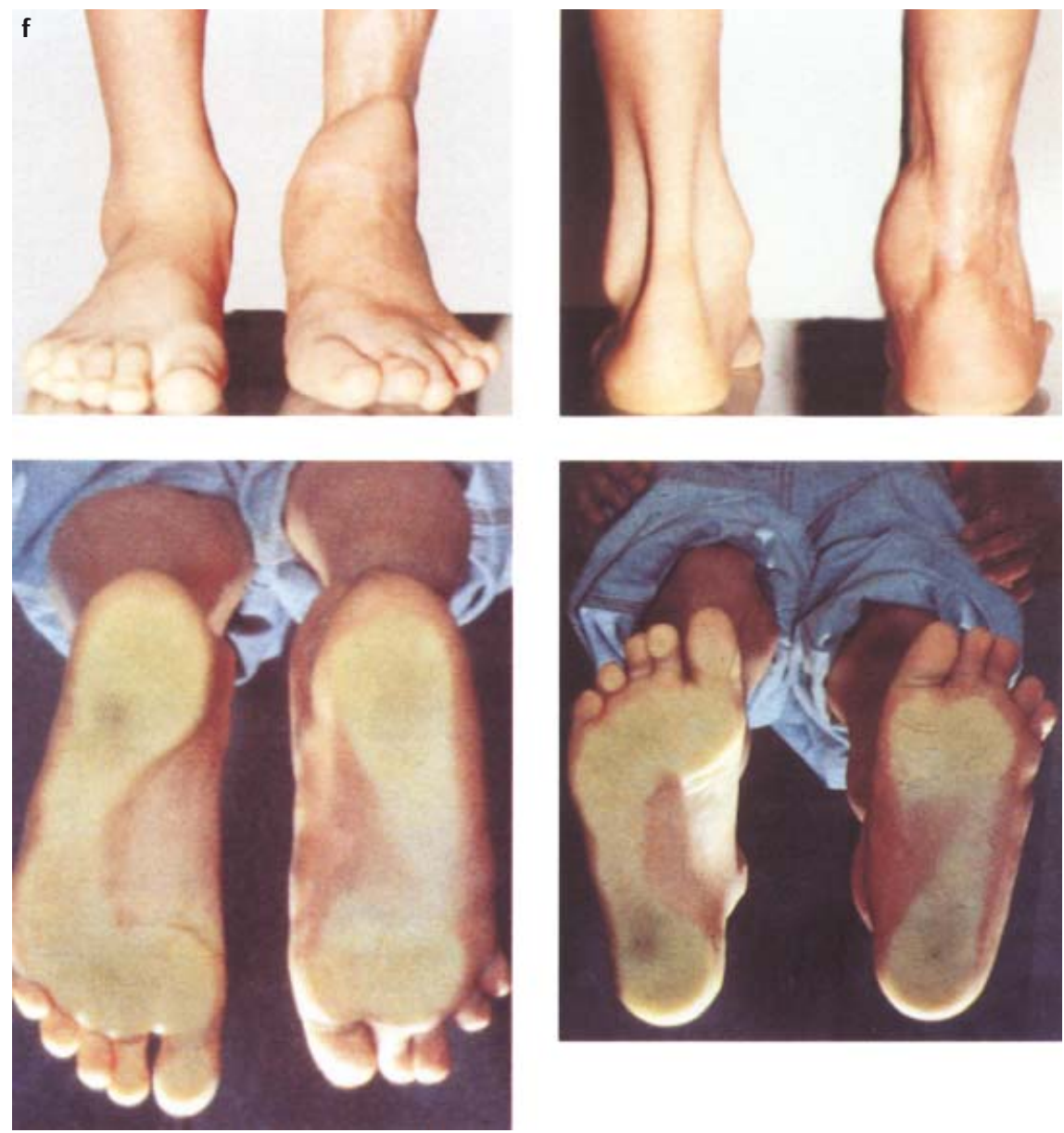

\section{Chopart/Lisfranc}

Bei posttraumatischer Verkürzung der lateralen oder medialen Fußsäule, insbesondere am noch wachsenden Fußskelett, können Osteotomien zum Beispiel am Calcaneus mit Verlängerung desselben (modifizierte Evans-Osteotomie) durch Einbringen eines autologen kortikospongiösen Spanes einen schweren posttraumatischen Pes plano valgus rekompensieren. Das Fallbeispiel 5 (Abb.5) zeigt, dass allein mit Korrekturosteotomien eine komplexe Fußfehlstellung, insbesondere am wachsenden Skelett auch nach schwerem Komplextrauma mit Fehlverheilung sehr wirksam sein können.

\section{Metatarsaleschaft}

Fehlverheilungen mit relevanter posttraumatischer Beschwerdesymptomatik sind in dieser Region sehr selten. Dies mag trotz der Häufigkeit dieser Frakturen mit $61 \%$ im Fußbereich [3] einerseits daran liegen, dass gröbere Fehlstellungen kaum vorliegen oder diese gut kompensiert werden können. Korrigierende Osteotomien werden erst dann notwendig, wenn Metatarsalgien von MT II/III auf eine Fehlverheilung von MT I oder von MT III/IV auf eine Fehlstellung von MT V zurückzuführen sind. Das heißt, dass MT I oder MT V aufgrund einer Fehlstellung nach dorsal nicht das übliche Maß des Körpergewichtes aufnimmt, sondern die benachbarten Metatarsalia zur Überbelastung gezwungen werden (Abb.6).

\section{Metatarsaleköpfchen}

Fehlverheilte subcapitale Metatarsalesowie Metatarsaleköpfchenfrakturen können rasch zur schwersten Metatarsalgie führen, weshalb die primäre korrekte operative Versorgung besondere Bedeutung hat. Frühkorrekturen nach stabiler oder instabiler Fehlverheilung mit Osteotomie und Achsenkorrektur sind seltene Indikationen, anspruchsvoll in Bezug zur Operationstechnik, aber seitens der Patienten dankbare Eingriffe (Abb. 7). 

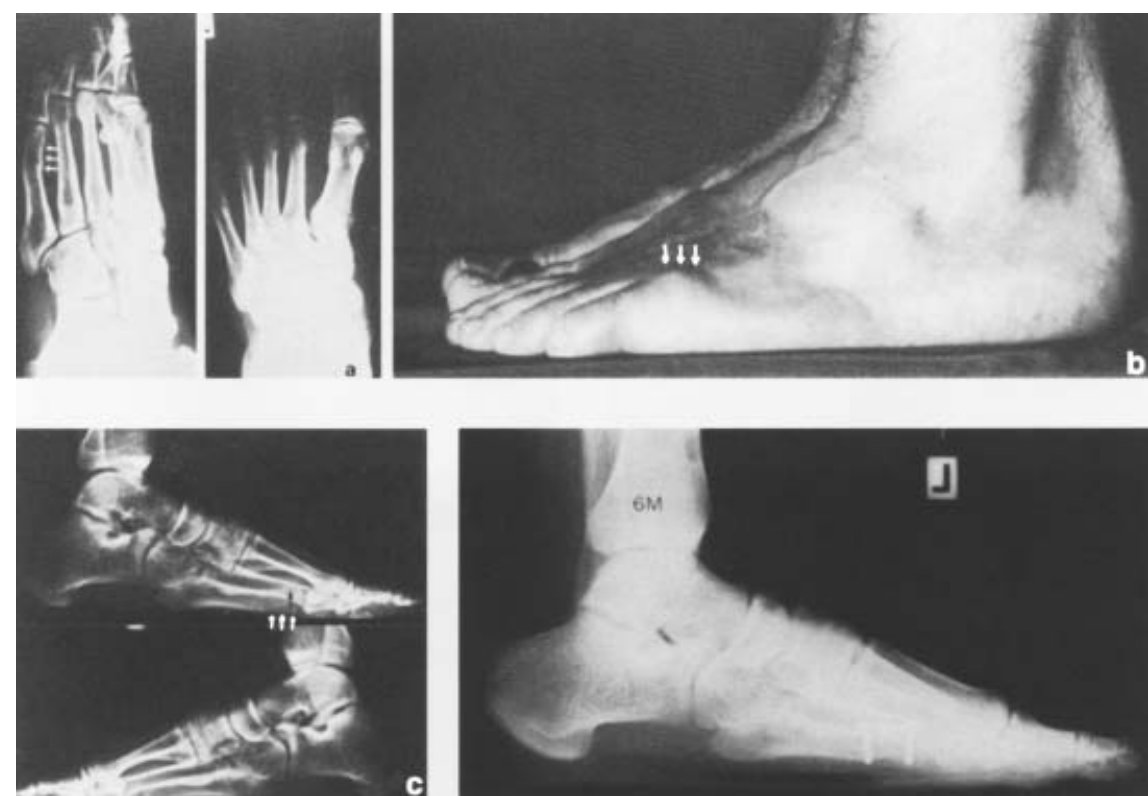

Abb. 6 Mechanisch bedingte Metatarsalgie: 25jähriger Patient, der sich 2 Jahre zuvor eine distale Metatarsale-V-Fraktur zuzog, die seinerzeit konservativ behandelt wurde. Wegen hartnäckiger Metatarsalgie-Beschwerden III/IV ist auswärts bereits eine Operation bei vermeintlicher Morton'scher Erkrankung durchgeführt worden. Wegen Persistenz der Beschwerden wird die Pathologie erst durch Belastungsaufnahmen deutlich. (a-c) Sowohl klinisch als auch radiologisch besteht eine deutliche Fehlverheilung des 5. MetatarsaleKöpfchens mit Kippung nach dorsal um annähernd $10^{\circ}$, so dass der biomechanisch wichtige 5. Strahl die Körperlast nicht ausreichend aufnehmen kann. Durch die insuffiziente 3Punkte-Belastung kommt es mechanisch bedingt zur Metatarsalgie, besonders am 4. Strahl. (d) Erst die flektierende Korrekturosteotomie kann die Biomechanik des Fußes wiederherstellen und zum Verschwinden der metatarsalgieformen Beschwerden führen.
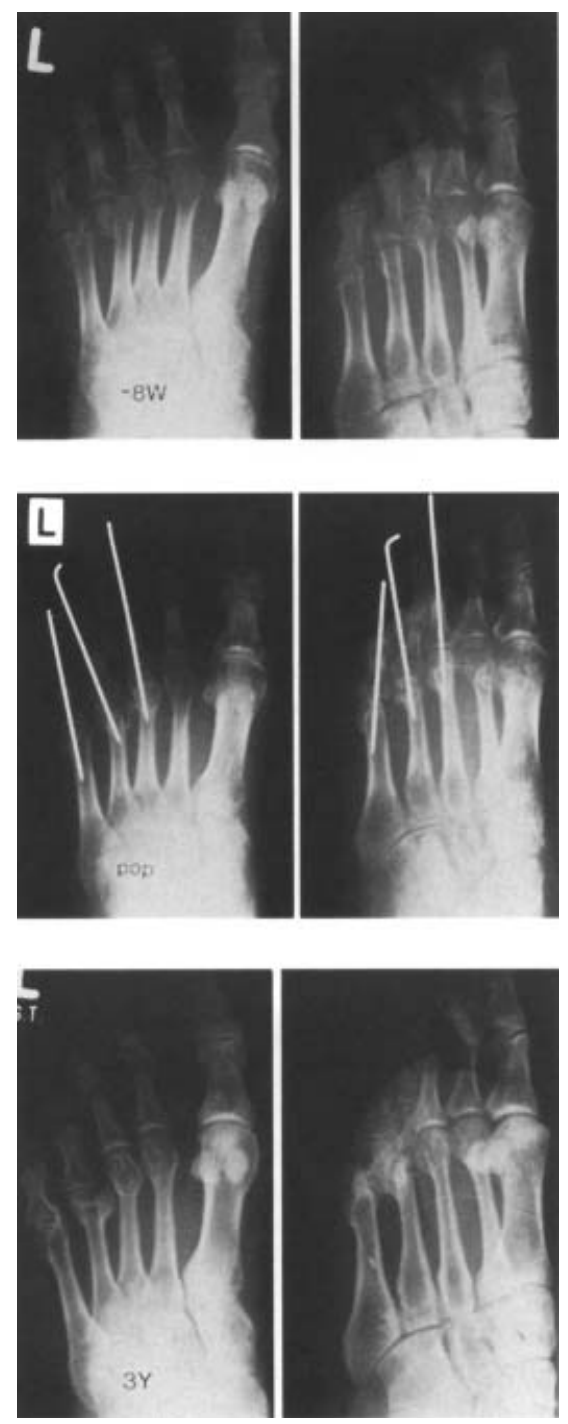

Abb. 7 Frühkorrektur fehlverheilter Metatarsaleköpfchen-Frakturen: 25jährige Patientin, die sich neben einer OSG-Luxationsfraktur subcapitale Metatarsale-Luxationsfrakturen III-V derselben Seite zuzog, die bei auswärtiger Behandlung erst nach 8 Wochen erkannt wurden. (a) Bei bestehender erheblicher Inaktivitätsosteoporose ist jetzt die operative Versorgung schwierig. Wegen erheblicher Schmerzhaftigkeit wird dennoch über eine dorsale Darstellung die offene Reposition, partielle Osteotomie und Rekonstruktion im Sinne der Frühkorrektur angestrebt. (b) Nach offener Einrichtung temporäre Spickdrahtfixation für 5 Wochen. (c) Die 3-JahresKontrolle zeigt eine gute Gelenkkongruenz mit nur minimaler Beschwerdesymptomatik durch den medialen Sporn am 5. Metatarsale.

\section{Literatur}

1 Dahlen C, Zwipp H. PC-basierte 3D-Software zur Operationsplanung in der rekonstruktiven Fußchirurgie. In: Hertel P, Rehm KE. Hefte zu Der Unfallchirurg, 63. Jahrestagung der Deutschen Gesellschaft für Unfallchirugie e.V., 17.-20. November 1999, Berlin: 1999; $343-344$

2 Pisani G. Trattato di chirurgia del piede. Edizioni Minerva Medica. Torino 1990

${ }^{3}$ Zwipp H, Ranft Th. Fehlverheilte kindliche Frakturen im Fußbereich. Orthop 1991; 20: $374-380$

4 Zwipp H. Chirurgie des Fußes. Springer Verlag Wien-New York 1994

Prof. Dr. med. H. Zwipp

Direktor der Klinik u. Poliklinik für

Unfall- $u$. Wiederherstellungschirurgie

Dr. med. C. Dahlen

Leitender Oberarzt

Dr. med. J. M. Gavlik

Oberarzt

Univ.-Klinikum Carl Gustav Carus

Fetscherstr. 74

01307 Dresden 